\title{
Criminologie
}

\section{La libération conditionnelle : le point de vue de la sociologie des organisations}

\section{Guy Lemire}

Volume 14, numéro 2, 1981

Libération conditionnelle : évolution et application (1899-1981)

URI : https://id.erudit.org/iderudit/017142ar

DOI : https://doi.org/10.7202/017142ar

Aller au sommaire du numéro

Éditeur(s)

Les Presses de l'Université de Montréal

ISSN

0316-0041 (imprimé)

1492-1367 (numérique)

Découvrir la revue

Citer cet article

Lemire, G. (1981). La libération conditionnelle : le point de vue de la sociologie des organisations. Criminologie, 14(2), 81-93. https://doi.org/10.7202/017142ar d'utilisation que vous pouvez consulter en ligne.

https://apropos.erudit.org/fr/usagers/politique-dutilisation/ 


\section{LA LIBÉRATION CONDITIONNELLE : LE POINT DE VUE DE LA SOCIOLOGIE DES ORGANISATIONS \\ Guy Lemire}

Il est étonnant de constater que, malgré le développement remarquable de la sociologie des organisations au cours des dernières décennies, la criminologie soit restée relativement imperméable à cette influence et n'ait pas été tentée d'utiliser davantage ces concepts et théories pour mieux comprendre le fonctionnement des appareils de l'administration de la justice pénale.

En fait, seul le milieu carcéral a pu bénéficier de cet apport, encore qu'il faille y apporter deux restrictions. La première est que certaines des études n'étaient pas à proprement parler criminologiques et ne touchent le milieu carcéral que de façon générale. Les recherches d'Etzioni* nous en fournissent un bel exemple et son mérite le plus remarquable à mon avis est d'avoir démontré comment, dans un premier temps, la présence ou l'absence de certaines caractéristiques appelait un modèle d'organisation carcérale corcitif ou normatif et, dans un deuxième temps, l'impact de ces modèles d'organisation sur tout programme de traitement. Toutefois, l'intérêt premier d'Etzioni était pour l'ensemble des organisations : aussi ne faut-il pas se surprendre que ses réflexions sur le milieu carcéral n'aient pas dépassé le niveau somme toute général.

La deuxième restriction nous vient des études plus proprement criminologiques, lesquelles ont fait du milieu carcéral leur principal sujet d'étude. Ces études constituent un apport majeur à la compréhension de l'organisation carcérale (2) et leur valeur demeure encore bien actuelle. Toutefois, ces textes datent de la première moitié des années ' 60 et il faut constater que notre compréhension de l'organisation carcérale n'a guère changé depuis ce temps.

Les réflexions sur les politiques pénales ont connu au cours de la dernière décennie un essor remarquable de telle sorte qu'à l'heure actuelle on est en mesure de critiquer les orientations des principaux agents de l'administration de la justice, de proposer des alternatives ou d'être carrément abolitionniste mais on sait bien

\footnotetext{
*Pour détails voir les ouvrages cités dans la bibliographie (1).
} 
peu de choses sur le fonctionnement de ces organisations. Quel qu'en soit le résultat, ces politiques ne pourront s'exprimer que dans une action collective ou une action organisée, selon les expressions de Crozier et Friedberg (3).

La compréhension de l'administration de la justice, essentielle en criminologie, nécessite une plus grande utilisation de la perspective «organisationnelle» et c'est cette dimension que le présent texte essaiera de mettre en valeur. En se détachant ainsi de la recherche criminologique actuelle, notre réflexion ne rejoint pas pour autant celle des administrateurs et des acteurs les plus influents de l'administration de la justice. Nous espérons en effet démontrer que les modèles d'organisation qu'ils utilisent, en misant surtout sur la rationalité, la logique du calcul ou la coordination mécanique, demeurent tributaires du modèle d'organisation formelle dont les insuffisances sont connues et démontrées depuis quelque temps déjà.

1. Il demeure en effet étonnant que le modèle d'organisation formelle soit encore si présent à l'heure actuelle. Même si la terminologie est maintenant plus raffinée, il ne fait aucun doute que division des tâches, contrôle hiérarchique, prise de décision demeurent des concepts clés des organisations bureaucratiques modernes et, pour reprendre l'expression d'Etzioni (4), on croit encore que ce qui est bon pour l'organisation est bon pour l'individu.

Crozier (5) a expliqué cette persistance en démontrant qu'une organisation bureaucratique n'arrive pas à se corriger en fonction de ses erreurs. L'illustration la plus claire de cette proposition nous est fournie par le milieu carcéral. Sykes (6) a bien démontré, dans son étude classique, comment la domination des gardes sur certains détenus était plus fictive que réelle et j'ai déjà eu l'occasion (7) d'expliquer qu'un directeur de pénitencier astucieux peut contrôler une population de détenus à travers quelques leaders à qui il accorde un statut privilégié. La socialisation ainsi réalisée auprès des détenus est donc celle de ces leaders, bien criminalisés, et il y a tout lieu de croire que les valeurs véhiculées n'ont rien à voir avec la réhabilitation. Le milieu carcéral, par son type d'organisation, favorise donc le renforcement des valeurs criminelles.

Même si cette démonstration ne date pas d'aujourd'hui, le milieu carcéral continue toujours à utiliser des modèles d'organi- 
sation formelle, hiérarchisés et de nature cœrcitive, tout en sachant fort bien que cela les empêche d'atteindre un de leurs objectifs.

Nous croyons que la réticence ou plus clairement, le refus des organisations à mettre de côté le modèle d'organisation formelle tient à un enjeu fondamental où s'affrontent liberté et contrôle. Le gestionnaire croit qu'il ne peut laisser la liberté des employés se manifester trop fortement et qu'il doit imposer des limites sous crainte de voir le đésordre s'installer. L'employé, d'autre part, aspire à la plus grande autonomie possible et n'a que faire de ces énormes quantités de directives et procédures qui lui sont imposées par des experts généralement éloignés des milieux d'exécution.

Les problèmes de l'organisation font donc appel à des concepts clés de notre société. Pour les criminologues, ce dilemme revêt d'autant plus d'importance qu'il est reproduit dans la relation ambiguë que nous entretenons avec les clients. Les criminologues auraient-ils tendance à reprendre avec les délinquants le même modèle de fonctionnement qu'ils ont développé avec leurs gestionnaires? Si tel était le cas, le conflit aide-contrôle ne s'expliquerait plus dans une perspective strictement clinique mais nécessiterait d'être situé dans une perspective d'organisation et deviendrait un problème de bureaucratie. Ou, comme dit Goffman (8) pour les institutions totalitaires : "Le fossé qui existe entre le personnel et les reclus est l'une des conséquences majeures du maniement bureaucratique d'importantes masses de gens.»

C'est notre conviction que l'organisation bureaucratique ne peut exercer le contrôle qu'elle souhaiterait et qu'elle permet l'expression de libertés imprévues et qui n'ont rien à voir avec l'efficacité de l'organisation. C'est ce que nous nous efforcerons maintenant de démontrer.

2. Le Service national des libérations conditionnelles (maintenant intégré au Service canadien des pénitenciers dans les Services correctionnels du Canada) a dicté dans un imposant manuel de procédures de près de 600 pages comment doit s'effectuer la surveillance des libérés conditionnels. Les règles et procédures constituent l'outil privilégié de l'agent et pour le S.N.L.C., un bon agent est celui ou celle qui connaît et applique les règles.

Pour s'assurer que ce manuel est bien suivi, le S.N.L.C. à mis sur pied un département de contrôle de la qualité : ce dernier s'assure que l'ex-détenu est bien rencontré aux périodes prescrites, 
que les mesures appropriées ont été prises et que les rapports rédigés couvrent bien les sujets demandés. L'agent qui satisfait ces normes est assuré d'un emploi à perpétuité! Les procédures sont devenues la préoccupation majeure des agents, ce qui signifie en d'autres termes que les moyens ont acquis une valeur en soi et sont devenus une fin. C'est ce que Merton (9) a appelé le déplacement des buts. Il en résulte des comportements rigides et ritualistes où l'on répète les mêmes gestes et où le centre d'intérêt n'est plus la réinsertion sociale du délinquant mais le respect du manuel de procédures.

Et pourtant ce manuel de procédures n'a pas toujours été aussi important. Au tout début de l'organisation, il était réduit à sa plus simple expression mais à chaque problème auquel il a fait face, le S.N.L.C. a répondu par de nouvelles règles qui ont mené à l'imposant document d'aujourd'hui. Dans ces conditions, il ne serait guère surprenant que d'ici cinq ans, il ait dépassé les 1000 pages!

Il est donc évident que le S.N.L.C. ne voit de solution à ses problèmes que dans le renforcement de ses procédures. C'est ce qui a fait dire à Crozier (5) que les erreurs de l'organisation ne servent qu'à la renforcer, de telle sorte que les règles deviennent de plus en plus nombreuses, tâchent de couvrir les plus petits détails et de parer au plus grand nombre possible d'éventualités.

À ces critiques, les gestionnaires répondent que les procédures ne sont que des lignes directrices qui doivent être complétées par le jugement de l'agent. Les agents ne croient pas à une telle explication car ils savent bien qu'en cas d'échecs d'une libération conditionnelle, ils seront pénalisés de ne pas avoir suivi les règlements à la lettre. Nous reviendrons plus loin sur la protection que retire l'agent du respect de ces procédures.

L'importance grandissante des procédures a comme corollaire, selon Crozier (5), la centralisation des décisions. Le modèle hiérarchisé administration centrale - région — district - bureau de section est bien connu. L'agent de libération conditionnelle n'est que l'exécutant de décisions prises à des niveaux supérieurs. Il est d'ailleurs significatif que le gestionnaire responsable de chaque bureau local ne soit plus comme avant un directeur de bureau, mais un responsable de secteur. En outre, l'agent doit exécuter les décisions d'un organisme parallèle, la Commission nationale des libérations conditionnelles. 
Crozier (5) dit à cet effet :

La centralisation est donc le second moyen d'éliminer le pouvoir discrétionnaire de l'être humain au sein d'une organisation. Le prix que l'organisation doit payer est celui d'une plus grande rigidité. Ceux qui décident ne connaissent pas directement les problèmes qu'ils ont à trancher; ceux qui sont sur le terrain et connaissent ces problèmes n'ont pas les pouvoirs nécessaires pour effectuer les adaptations et pour expérimenter les innovations devenues indispensables.

Le S.N.L.C. se présente donc comme une organisation axée sur les procédures et règles, avec un processus de décision centralisé et hiérarchisé. C'est pourquoi on y observe des comportements rituels et rigides, peu portés au changement. Même si ce n'est pas l'objet du présent texte, ceci pourrait expliquer pourquoi le S.N.L.C. a opposé tant de résistance aux nouvelles définitions de rôles qu'amenait son intégration au Service canadien des pénitenciers.

Dans cet essor des procédures et cette complexité des prises de décisions, il y a, à n'en pas douter, un effort de rationalité pour contrôler le plus possible la liberté des agents et créer une organisation à l'image de ses dirigeants. À première vue, on pourrait facilement conclure que ces derniers ont réussi et ce n'est certes pas au niveau des écritures qu'on pourra les prendre en défaut. Là où le bât blesse, même au niveau formel, c'est lorsqu'on se rend compte que les procédures sont devenues la véritable finalité de cette organisation, que ces procédures reflètent surtout le désir de contrôler les libérés conditionnels et que la relation d'aide n'est somme toute qu'un bénéfice marginal. Sur les 600 pages de procédures, combien sont consacrées à l'aide à apporter au libéré conditionnel? Est-il exagéré de dire qu'il sera de plus en plus difficile de distinguer l'agent de libération conditionnelle d'un agent de police? Après avoir tant investi au niveau du contrôle il serait difficile d'en être autrement.

Il serait toutefois malvenu de considérer les agents de libérations conditionnelles comme des victimes étouffées sous les contrôles et privées de liberté.

3. On serait même tenté de croire que le système actuel leur convient davantage qu'ils ne veulent bien le laisser paraître. Si la situation est telle que nous venons de la décrire, deux choix s'offrent à eux : ou bien ils quittent le S.N.L.C. ou bien ils se révoltent. Or, ils ne font ni l'un ni l'autre. 
Nous nous efforcerons donc, au cours des prochains paragraphes, de démontrer comment les agents utilisent les mécanismes formels de procédures et de centralisation pour se protéger et «développer des réseaux informels de pouvoir où s'exprime leur liberté » $(5)$.

Nous avons mentionné plus tôt qu'en respectant les règles et procédures, l'agent de libération conditionnelle s'assure une protection à toute épreuve vis-à-vis ses supérieurs. C'est là un avantage non négligeable du modèle d'organisation formelle parce qu'il augmente l'indépendance de l'employé vis-à-vis son employeur.

Nul n'est mieux placé que le responsable de secteur pour comprendre cette situation : les règles venant d'en haut, la seule autorité qui lui reste c'est le contrôle pous s'assurer qu'elles sont respectées. Et encore là, cette autorité est mitigée puisqu'en fin de compte ce sont les agents régionaux de contrôle de la qualité qui iront vérifier en détail le travail de l'agent. Le tableau se présente donc ainsi : en satisfaisant aux exigences des agents régionaux, l'agent n'a en définitive plus de compte à rendre à son supérieur immédiat, le responsable de secteur. Ainsi ce dernier agit davantage comme entremetteur ou agent d'information que comme supérieur. S'il se sent une âme de gestionnaire, il sera insatisfait de cette situation; s'il aime la sécurité, il appréciera son confort.

Non seulement l'agent de libération conditionnelle se sent-il plus indépendant de ses supérieurs grâce à son adhésion aux règles, mais il se sent également plus en sécurité vis-à-vis les libérés conditionnels et il ne manque pas de le faire savoir à ceux-ci : "Ce sont les règlements, moi je n'y peux rien, » Voilà une phrase que tout libéré conditionnel a certes entendu plus d'une fois!

La centralisation des décisions contribue également à ce sentiment de sécurité : "C'est une directive de la Commission »... « Le Manuel des procédures est imposé par Ottawa »... «Ca vient de la région. » Que peut répliquer l'ex-détenu à un tel argument? L'honneur de l'agent est sauf!

Et c'est ici qu'apparaît le caractère dysfonctionnel du S.N.L.C. Dans la mesure où l'adhésion aux règlements a priorité, le service à la clientèle passe en second lieu. Et ceci, ce n'est pas uniquement la relation d'aide qui en est cause, c'est également la dimension de contrôle. En effet, on pourrait penser que les procédures et la centralisation servent à mieux contrôler le libéré con- 
ditionnel. Que ce dernier doive rencontrer l'agent deux fois par mois, qu'il doive prouver qu'il travaille ne solutionnent en rien le problème du contrôle du libéré conditionnel. Il peut satisfaire ces normes et avoir des activités délinquantes.

Les normes ont donc priorité mais ne règlent rien et l'exdétenu qui joue le jeu le sait bien. Il apprend à se plier devant les exigences organisationnelles et il y a fort à parier qu'il y acquiert une certaine aisance. Nous serions tenté de conclure cette partie en disant que, dès qu'il accepte de jouer le jeu des procédures et de la centralisation, l'agent s'impose des embûches qui rendront difficile, sinon impossible, une véritable relation avec le libéré conditionnel. L'agent se retrouve alors aux antipodes de l'objectif qu'il est censé poursuivre sur le plan professionnel. C'est en ce sens qu'il y a dysfonction.

4. Nous avons tenté de démontrer dans les paragraphes précédents que l'agent trouve un certain profit à utiliser le modèle d'organisation bureaucratique pour se protéger à la fois de ses supérieurs et de ses clients. Ce n'est pas la seule faille de ce modèle.

L'indépendance relative acquise par les agents est en relation directe avec l'autonomie et l'isolement de chaque bureau. Comme dit Crozier (5) : «Une organisation bureaucratique de ce type va se trouver composée d'une série de strates superposées, communiquant très peu entre elles. » Il est bien connu que les communications, par exemple, entre le bureau de secteur et la direction régionale sont restreintes à des mémorandums et directives, et se font généralement à sens unique, du haut vers le bas.

Mais c'est surtout avec la Commission nationale des libérations conditionnelles que l'isolement des agents porte à conséquence. Il empêche l'individualisation de l'intervention auprès du délinquant : c'est ainsi que la Commission applique de façon de plus en plus automatique un régime de libération progressive qui sert plus des fins technocratiques que des besoins réels. L'exdétenu doit presque obligatoirement séjourner dans une maison de transition avant d'obtenir une libération conditionnelle totale et ce, même s'il n'a pas besoin d'une telle maison et même si l'agent sait qu'il n'en a pas besoin.

Il est possible dans ces circonstances, que l'on assiste à un essor des maisons de transition et ça pourrait passer à première vue comme une mesure progressiste qui diminue la période d'in- 
carcération, alors qu'en réalité ça ne résulterait que de l'utilisation exagérée d'une mesure qui n'est somme toute utile qu'à une certaine catégorie de délinquants. Cela constituera un bel exemple du principe qui établit qu'une organisation utilise ses dysfonctions pour se mieux renforcer et même se développer.

Cet isolement, on le perçoit bien, aura comme conséquence de permettre aux agents de chaque secteur de devenir de plus en plus « auto-suffisants». Puisque les procédures, la centralisation et le manque de communication rendent difficile le service individualisé à la clientèle, l'isolement transporte l'intérêt des agents vers le groupe de paris dont ils font partie. Le groupe prend alors une importance capitale dans le travail des agents : c'est ainsi qu'on assiste aux pressions, à l'émergence du leadership informel, aux conflits. L'agent n'est donc plus contrôlé uniquement par des procédures mais également par le groupe. Il lui sera difficile, et quelquefois pénible, d'exprimer son individualité. La pression du groupe devient en fait le principal instrument de contrôle. C'est la loi du milieu! Quel agent oserait dépasser la norme prescrite production d'enquête communautaire même s'il était convaincu que cette norme est trop peu élevée?

La vie du groupe et sa survivance deviennent des fins en soi. Dans cette optique, le changement ne peut être que menaçant car il risque de perturber l'équilibre du groupe. Bien sûr, cette domination du groupe comporte des aspects nettement positifs : l'esprit de corps et la solidarité en sont les deux manifestations les plus éclatantes. Ceci peut contribuer à expliquer l'apparente dichotomie qui est souvent notée lors de conversations avec les agents : d'une part, l'évidente insatisfaction vis-à-vis leur travail et d'autre part "la satisfaction de travailler avec l'équipe de ce secteur». Et c'est parce que l'équipe est une fin en soi que son évaluation peut être aussi clairement distincte de celle des tâches accomplies. Si la vie de groupe n'était qu'un des moyens utilisés pour accomplir les tâches d'agent (ce qu'il devrait être en réalité), l'insatisfaction des agents vis-à-vis celles-ci dominerait tout le reste. Ce n'est pas le cas et ces deux réalités semblent cœxister pacifiquement!

On comprendra que cette domination du groupe mène directement au développement de pouvoirs parallèles. Ce phénomène est déjà bien connu dans certains secteurs de l'administration de la justice et on a déjà vu un directeur de police accuser le syndicat de constituer un pouvoir parallèle. Ce n'est pas le fruit du hasard que 
ce phénomène se soit développé dans un milieu très hiérarchisé et centralisateur (le parallèle est intéressant avec le milieu carcéral à sécurité maximale où certains comités de détenus ont tenu tête à la direction de ces institutions).

Ce phénomène n'a pas été étudié dans les bureaux des agents de libération conditionnelle : aussi est-il difficile d'en parler avec précision. Toutefois, il y a fort à parier que, à une époque où le contrôle des libérés conditionnels domine nettement la relation d'aide, les agents qui expriment ou concrétisent le mieux cette dimension de contrôle soient également ceux qui détiennent le leadership informel. Dans ces conditions, il ne serait pas surprenant de voir de simples agents détenir plus de pouvoirs que le responsable du secteur.

5. Il est quand même ironique qu'un modèle d'organisation formelle dont le souci de contrôle est si évident, donne naissance à des manifestations non formelles qu'il n'est pas en mesure de contrôler. Mais ce n'est pas ce qui doit inquiéter le plus. L'inquiétude doit venir du fait que les moyens ont remplacé les fins, que les agents sont à la fois protégés par et désengagés vis-à-vis ce système et que le libéré conditionnel devient une préoccupation secondaire. En fin de compte, l'agent de libération conditionnelle travaille peu au niveau de la relation d'aide et ne peut être un bon policier. Il n'est donc pas surprenant de constater que les agents croient de moins en moins en se qu'ils font et que la Commission soit en perte de crédibilité. Les nominations contestées de certains commissaires viennent tout simplement confirmer qu'il n'est plus nécessaire d'avoir une certaine compétence pour bien fonctionner au sein de ces organismes mais qu'il suffit tout simplement d'appliquer des normes bureaucratiques rigides de façon rituelle.

La C.N.L.C. et le S.N.L.C. sont en perte de vitesse parce qu'ils ont perdu leur spécificité : un ordinateur pourrait rendre les décisions de façon plus stable et cohérente tandis qu'un bon policier pourrait contrôler le délinquant avec autant d'efficacité que ne le fait l'agent à l'heure actuelle. Dans ces conditions, il serait facile de conclure que la libération conditionnelle devrait être abolie. Cette conclusion m'apparaît toutefois erronée parce qu'elle confond la mesure de réinsertion sociale avec le modèle d'organisation dans lequel elle est vécue. Ce n'est pas la libération conditionnelle qui doit être remise en question mais bien le modèle 
d'organisation formelle, hiérarchisé et centralisateur, dans lequel on l'a confirmée. Je crois qu'il y aura toujours place pour une aide individualisée à l'ex-détenu. Que l'on considère ce dernier handicapé soit par son appartenance à une classe soit par ses traits de personnalité, on le maintient dans cet état d'infériorité si on n'est pas prêt à mettre en place les mécanismes susceptibles de l'aider à s'en sortir.

Même si le but du présent article était de poser le problème de la libération conditionnelle dans ce qui me semble sa juste perspective et que les limites d'espace ne nous permettent pas de trop le prolonger, j'aimerais tout de même indiquer des éléments de solution.

En premier lieu, il faut absolument mettre fin à ce que Crozier appelle dans son dernier livre, "Le délire des procédures 》 (10). Nous avons indiqué comment cette insistance exagérée sur les règles a comme conséquence de créer des dysfonctions. Elle empêche également une individualisation de l'intervention auprès du délinquant. Il n'y a pas d'autre choix que de revenir à un minimum de règlements et à un maximum d'initiative de la part des agents. On ne peut créer de situations de responsabilité, tant chez l'agent que chez le délinquant, en maintenant les gens dans un carcan.

En second lieu, les différents acteurs du système doivent entreprendre une révision de leur fonctionnement qui risque d'être pénible si on rappelle que le modèle d'organisation actuel les protège si bien. Et c'est là le nœud du problème. Les agents oseront-ils sacrifier une partie de leur sécurité actuelle au profit de l'efficacité? Aura-t-on le courage d'affirmer et de prouver que la compétence professionnelle est plus importante que l'habileté technocratique? Ces choix ne sont certes pas faciles à faire mais ils sont devenus inévitables si on veut se libérer du bourbier actuel.

La direction, quant à elle, doit restreindre le plus possible un modèle d'organisation basée sur la hiérarchie, le contrôle et la centralisation. Plus les décisions sont prises à des niveaux qui s'éloignent de la réalité, moins ces décisions risquent d'être adéquates. Une délégation d'autorité implique donc une nouvelle approche pour la direction, moins centrée sur le contrôle et les règlements, et plus orientée vers l'animation et la distribution des ressources. 
En troisième lieu, le Service correctionnel du Canada (S.C.C.) devra se donner un modèle d'organisation compatible avec les principes d'action qu'il s'est donnés. Il y a quelques années, le S.C.C. affirmait que le délinquant est le principal responsable de sa criminalité et de sa réinsertion sociale (11). Un document plus récent, mais encore confidentiel au moment de la rédaction de cet article, indique que l'on se dirige vers un modèle de responsabilité partagée entre le délinquant, le S.C.C. et la société. Dans cette optique, il faut souligner que le S.N.L.C. fait des efforts louables pour impliquer la communauté dans le processus correctionnel.

Toutefois, le type d'organisation corcitive qu'a conservé le S.C.C. est incompatible avec une prise de responsabilités de la part des délinquants. On ne peut baser son intervention sur la crainte, la menace et la dépendance d'une part, et espérer un comportement adulte et responsable d'autre part. Etzioni (1) a bien démontré que ce type d'organisation aboutit généralement à un engagement aliénatif chez ses membres, engagement qui est à l'opposé de l'adhésion désirée. Si le délinquant doit être responsable de sa réinsertion sociale, cela implique qu'il soit placé en situation de responsabilité avec le pouvoir et l'autorité que cela implique. On ne peut maintenir le délinquant dans un état de dépendance et le placer dans des situations où il n'a que des privilèges d'une part, et continuer à parler de ses responsabilités d'autre part. Sinon ce principe de responsabilité du délinquant n'aura été qu'une vulgaire stratégie bureaucratique destinée à détourner l'attention de la véritable responsabilité du Service correctionnel du Canada.

Nous nous retrouvons donc au cœur de ce que j'ai appelé au début de l'article le véritable enjeu de l'organisation : liberté et contrôle. L'organisation correctionnelle a toujours misé sur le contrôle au détriment de la liberté et son utilisation du modèle de l'organisation formelle le reflète bien. J'espère avoir démontré que cette tentative exagérée de contrôle n'empêche pas l'expression de libertés non formelles, souvent dysfonctionnelles. Il serait peutêtre temps d'investir dans la liberté des membres de l'organisation pour obtenir un meilleur équilibre. En affirmant la responsabilité du délinquant, le S.C.C. n'a guère d'autre alternative.

6. Le présent texte avait pour but de présenter une nouvelle perspective qui permette de mieux comprendre les problèmes de la libération conditionnelle. Je crois qu'une partie des difficultés 
rencontrées dans l'implantation de mesures d'intervention auprès des délinquants ne provient pas de la qualité ou de l'opportunité des dites mesures, mais du contexte "organisationnel 》 dans lequel elles doivent être vécues. Le modèle d'organisation formelle, surtout préoccupé par le contrôle hiérarchique (même avec ses raffinements modernes de planifications, de gestion par objectif, etc.) m'apparaît difficilement compatible avec la véritable relation d'aide. C'est toute la signification que me semble contenir le texte de Goffman (8), cité au début, lorsqu'il affirme que le fossé entre le personnel et le reclus est la conséquence du maniement bureaucratique des gens. Cela m'apparaît d'autant plus vrai que l'employé a vis-à-vis de la direction des sentiments semblables à ceux que le délinquant a vis-à-vis ce même employé. Il serait intéressant de mettre en parallèle les sentiments du libéré conditionnel vis-à-vis l'agent et ceux de l'agent vis-à-vis sa haute direction. Je suis persuadé que les différences seraient minimes et c'est fondamentalement un problème d'organisation. Dans ces conditions, je serais tenté de dire qu'on a imputé aux tenants de la perspective clinique des échecs dont ils n'étaient pas responsables. Ceci est évident dans le milieu carcéral mais c'est le même problème qui guette les bureaucraties professionnelles en milieu ouvert (qu'on pense aux difficultés de la loi 24).

Dans cette optique, le dilemme contrôle-relation d'aide ne peut plus être considéré comme une question strictement clinique mais doit être situé dans la perspective plus vaste de toute action organisée qui est un mélange plus ou moins harmonieux de contrôle et de liberté. Faut-il se surprendre que, dans des organisations où le contrôle est omniprésent, la relation d'aide soit inefficace? Plutôt que de s'acharner à transformer ou même détruire les interventions cliniques, il serait peut-être temps de s'attaquer aux organisations qui neutralisent ces interventions.

Les modèles formels et cœrcitifs ont été trop longtemps considérés comme des faits acquis dans le domaine de l'administration de la justice. Pourtant, on voit apparaître un peu partout dans la société des tentatives de plus en plus nombreuses de prises en charge et de récupérations par les citoyens de leurs situations. Pendant ce temps, les professionnels préfèrent la sécurité à l'innovation.

Le temps est peut-être venu pour les experts de suivre la voie tracée par des citoyens. 


\section{BIBLIOGRAPHIE}

1) ETZIONI, A. (1965), aOrganizational Control Structure $\gg$ in March J.G. (Ed.) Handbook of Organizations, Chicago, Rand, McNally \& Co.

ETZIONI, A. (1975). A Comparative Analysis of Complex Organizations. New York. Free Press.

2) CLOWARD, R. et al. (eds.) (1960). Theoretical Studies in the Social Organization of the Prison. New York. Social Science Research Council.

CRESSEY, D. (1961). The Prison: Studies in Institutional Organization and Change. New York. Holt, Rinehart and Winston.

CRESSEY, D. (1965). a Prison Organizations * in March, J.G. (Ed.). Handbook of Organizations, Chicago. Rand, McNally \& Co.

3) CROZIER, M.; FRIEDBERG, E. (1977). L'acteur et le système. Paris. Éditions du Seuil.

4) ETZIONI, A. (1971). Les organisations modernes. Gembloux (Belgique : Ducolot.

5) CROZIER, M. (1963). Le phénomène bureaucratique. Paris. Éditions du Seuil.

6) SYKES, G. (1958). Society of Captives. Princeton, N.J. Princeton University Press.

7) LEMIRE, G. (1979). *Évolution de la criminalité et nouveaux types de clientèle carcérale 》 in Le rôle et la formation des agents d'unité résidentielle, Montréal. Solliciteur général.

8) GOFFMAN, E. (1968). Asiles. Paris. Éditions de Minuit.

9) MERTON, R.K. (1940). \& Bureaucratic Structure and Personality », in Social Forces, XVIII, p. 560-568.

10) CROZIER, M. (1980). Le mal américain. Paris. Fayard.

11) GROUPE DE TRAVAIL SUR LA CRÉATION DU SERVICE CANADIEN DE CORRECTION INTÉGRÉ (1977). Le rôle des services correctionnels fédéraux au Canada. Ottawa. Solliciteur général. 